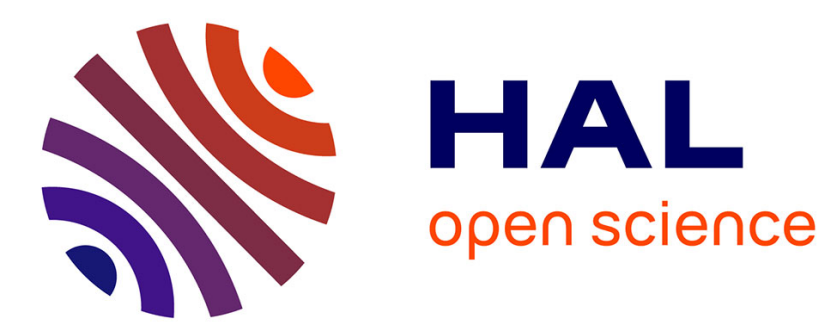

\title{
Orientational disorder in plastic neopentane (paralipomena)
}

Ph. Depondt, M. Debeau

\section{To cite this version:}

Ph. Depondt, M. Debeau. Orientational disorder in plastic neopentane (paralipomena). Journal de Physique, 1987, 48 (9), pp.1513-1519. 10.1051/jphys:019870048090151300 . jpa-00210583

\section{HAL Id: jpa-00210583 https://hal.science/jpa-00210583}

Submitted on 1 Jan 1987

HAL is a multi-disciplinary open access archive for the deposit and dissemination of scientific research documents, whether they are published or not. The documents may come from teaching and research institutions in France or abroad, or from public or private research centers.
L'archive ouverte pluridisciplinaire HAL, est destinée au dépôt et à la diffusion de documents scientifiques de niveau recherche, publiés ou non, émanant des établissements d'enseignement et de recherche français ou étrangers, des laboratoires publics ou privés. 
Classification

Physics Abstracts

61.12

\title{
Orientational disorder in plastic neopentane (paralipomena)
}

\author{
Ph. Depondt and M. Debeau \\ D.R.P. (LA 71), Université Pierre et Marie Curie, 4, place Jussieu, Tour 22, 75252 Paris Cedex 05, France
}

(Reçu le 6 mars 1987, accepté le 13 mai 1987)

\begin{abstract}
Résumé. - Des expériences de diffraction de neutrons effectuées sur des monocristaux de néopentane $\left(\mathrm{C}\left(\mathrm{CD}_{3}\right)_{4}\right)$ sont réinterprétées à l'aide d'un modèle TLS adapté afin de rendre compte autant que possible du désordre d'orientation. L'on obtient ainsi que les molécules sont localisées autour de leur orientation la plus probable à toutes les températures ; les variables orientationnelles montrent une dispersion importante mais pratiquement indépendante de la température, sans manifestation apparente de la dynamique réorientationnelle. Au contraire, les déplacements des centres de masse, toujours nettement plus grands que ce que l'on attendrait d'une contribution de phonons, s'accroissent fortement à haute température.
\end{abstract}

\begin{abstract}
Neutron diffraction experiments performed on orientationally disordered neopentane $\left(C\left(C D_{3}\right)_{4}\right)$ single crystals are interpreted using a TLS model adapted in order to account as well as possible for the orientational disorder. The molecules are found to be always localized around their most probable orientations at every temperature ; the orientational variables display a large, but practically temperature-independent, dispersion with no sign of reorientational dynamics. In contrast, the centre-of-mass displacements, always much larger than what one expects from the phonon contribution, strongly increase at high temperatures.
\end{abstract}

\section{Introduction.}

Neopentane $\left(\mathrm{C}\left(\mathrm{CD}_{3}\right)_{4}\right)$ exhibits an orientationally disordered phase between $140 \mathrm{~K}$ and $256.5 \mathrm{~K}$ in which the molecular centres of mass are set on a f.c.c. lattice while the orientations of the molecules are disordered, a situation often referred to as a plastic crystal or ODIC (Orientational Disorder In Crystals). Coherent diffraction experiments can provide structural information about this disorder which can be described by $P(\Omega)$, the orientational probability density function or o.p.d.f. The present article follows a previous article [1] in which neutron diffraction experiments had been interpreted in terms of an expansion of $P(\Omega)$ on a basis of symmetry adapted functions [2] (Appendix 1). It has been established that the tetrahedral molecules were most often oriented so that one $\overline{4}$ molecular symmetry axis is parallel to one fourfold crystal axis, while the other two are parallel to two twofold crystal axes. This situation, called $D_{2 \mathrm{~d}}$, offers six equivalent, but physically distinct, orientations with respect to the crystal reference frame.

However, the amount of data supplied by diffraction experiments, i.e. the number of non-equivalent
Bragg peaks for which intensity measurements are possible, is very limited in orientationally disordered crystals. Indeed, the Debye Waller factor related to the molecular centre-of-mass translations is very large, causing a rapid decrease of the intensity as the scattering angle increases. Consequently, the number of adjustable parameters in any model can hardly be much larger than 5 or 6 , resulting in the fact that the expansion of $P(\Omega)$ had to be truncated at a fairly low order, leading to a relatively smooth function, so that the maxima of the o.p.d.f. obtained through that analysis would be blurred by large truncation effects.

We have thus reanalysed our data following a different procedure : assuming the now well established $D_{2 \mathrm{~d}}$ situation, we have used the classical TLS model $[3,4]$ around each of the equilibrium positions. The results of this new study are reported below and show that, in the present case, a good physical insight can be obtained within the TLS description.

\section{Model.}

In order to reduce the number of adjustable parameters (in principle three Debye Waller factors per 
atom), the TLS model assumes rigid molecules $\left({ }^{1}\right)$ which perform translational and librational motions around an equilibrium position. The translational motion is accounted for by $T_{i j}=\left\langle u_{i} u_{j}\right\rangle$, the librational motion by $L_{i j}=\left\langle\theta_{i} \theta_{j}\right\rangle$ and the correlations between the centre-of-mass translations and the librations by $S_{i j}=\left\langle u_{i} \theta_{j}\right\rangle$, hence the mnemonic TLS. In these definitions, $u_{i}$ is a displacement parallel to the $i$ axis $(i=x, y, z)$ of the molecular centre-of-mass from its central position, while $\theta_{i}$ stands for a rotation around the $i$ axis, and $\langle>$ denotes a thermal average.

Considering one neopentane molecule in a $D_{2 \mathrm{~d}}$ orientation, such that one $\overline{4}$ molecular axis is parallel to $z$ while the other two are parallel to $x+y$ and $x-y$ (Fig. 1), the non-zero parameters of the model reduce, for this molecule, to [4] :

$$
\begin{gathered}
\left\langle u_{x}^{2}\right\rangle=\left\langle u_{y}^{2}\right\rangle \\
\left\langle u_{z}^{2}\right\rangle \\
\left\langle\theta_{x}^{2}\right\rangle=\left\langle\theta_{y}^{2}\right\rangle \\
\left\langle\theta_{z}^{2}\right\rangle \\
\left\langle u_{x} \theta_{y}\right\rangle=\left\langle u_{y} \theta_{x}\right\rangle .
\end{gathered}
$$

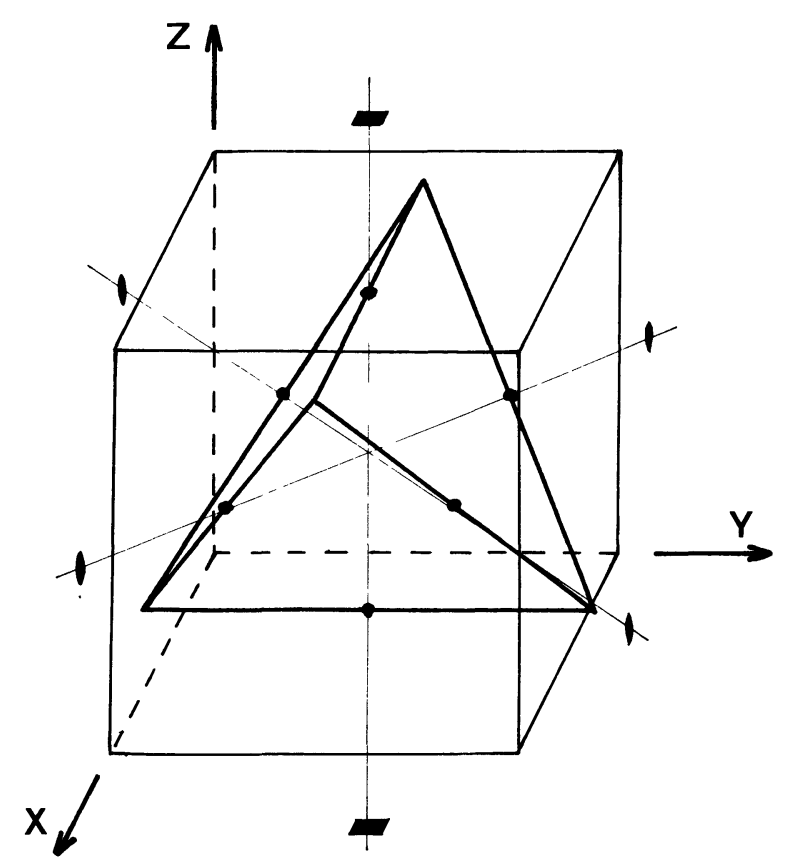

Fig. 1. - Tetrahedron with a $D_{2 \mathrm{~d}}$ orientation in a cube. One tetrahedric $\overline{4}$ axis is parallel to $z$, one of the cubic fourfold axes. $x$ and $y$ are the other two cubic 4 fold axes. The two remaining tetrahedric $\overline{4}$ axes are parallel to two cubic twofold axes $x+y$ and $x-y$. The solid circles show the intersections of the symmetry axes with the edges of the tetrahedron.

$\left.{ }^{1}\right)$ The intramolecular methyl group reorientations have been ignored since, as the corresponding barriers are high $\left(\simeq 18 \mathrm{~kJ} \mathrm{~mol}^{-1}\right.$ ) [12], the proportion of reorienting methyl groups is small.
Following for instance reference [3], this provides a diffraction structure factor $F_{1}^{\mathrm{TLS}}(q)$.

The six physically distinct $D_{2 \mathrm{~d}}$ orientations for the molecule in the disordered phase may be obtained through permutations of the crystal axes, giving $F_{j}^{\mathrm{TLS}}(q)(j=1,6)$. The structure factor, within the TLS approximation, is thus the sum of six terms :

$$
F^{\mathrm{TLS}}(q)=\frac{1}{6} \sum_{j=1,6} F_{j}^{\mathrm{TLS}}(q) .
$$

Equation (2) accounts for molecules in, or close to, the $D_{2 \mathrm{~d}}$ orientations which were assumed to be the starting point for the model. However, if, as expected, the molecules perform frequent reorientations from one $D_{2 \text { d }}$ orientation to another, many molecules should be in between these orientations at a given time. The simplest way to describe this is an isotropic structure factor :

$$
\begin{array}{r}
F_{\text {iso }}(q)=\exp \left(-\frac{1}{2} q^{2}\left(\frac{2\left\langle u_{x}^{2}\right\rangle+\left\langle u_{z}^{2}\right\rangle}{3}\right)\right) \times \\
\times \sum_{i=0}^{2} n_{i} f_{i} \frac{\sin \left(q r_{i}\right)}{q r_{i}}
\end{array}
$$

where $i$ labels the three shells of the $\mathrm{C}\left(\mathrm{CD}_{3}\right)_{4}$ molecule, namely :

0 corresponds to the central carbon atom, 1 to the four methyl carbon atoms and 2 to the twelve deuterium atoms; $r_{i}$ is the radius of shell $i$; $f_{i}$ is the coherent diffusion length of atoms on shell $i$; and $n_{i}$ is the number of atoms on that shell.

The final structure factor can be written as the weighted sum of the TLS factor and the isotropic contribution :

$$
F(q)=F_{\text {norm }}\left((1-p) F^{\mathrm{TLS}}(q)+p F_{\text {iso }}(q)\right)
$$

where $p$ is the proportion of isotropy and $F_{\text {norm }}$ a normalisation factor which is proportional to the volume of the sample.

The nine adjustable parameters of the model are the five TLS parameters, $F_{\text {norm }}, p, r_{1}$ and $r_{2}\left(r_{0}=0\right)$.

\section{Results.}

A first series of fits was performed with all parameters free to adjust. These showed that $p$ goes to zero at all temperatures; it was then fixed at zero without changing the result. The shell radii were fixed at an average value $\left(r_{1}=1.57 \AA\right.$ and $r_{2}=$ $2.099 \AA$ ) so that the results at different temperatures could be compared. It was verified that this did practically not alter the quality of the fits. The values for $r_{1}$ and $r_{2}$ are smaller than in reference [1] (1.58 $\AA$ and $2.17 \AA$ ) because of the apparent shortening [3] of the bond lengths due to librational motion. No correction with temperature of the bond lengths was necessary as $\left\langle\theta_{x}^{2}\right\rangle$ and $\left\langle\theta_{z}^{2}\right\rangle$ change very little as the temperature increases. 
At the lowest temperature, $T=150 \mathrm{~K}$, the cell parameter was found to be : $a=8.56 \pm 0.13 \AA$, a smaller value than one would expect if one assumes a linear variation of the cell parameter with temperature. This point was further explored : a fit was performed with $a=8.63 \AA$, the value it would have in the linear case. This lies well within the error bar for the cell parameter but might however affect the fit calculations. The result is that the quality of the fit decreases slightly $(R W=8 \% v s .7 .5 \%)$ while the parameters including $r_{1}$ and $r_{2}$ are not much affected. The choice of a given value for $a$ is therefore not critical.

The calculation then ends up with a six-parameter fit $\left(F_{\text {norm }},\left\langle u_{x}^{2}\right\rangle,\left\langle u_{z}^{2}\right\rangle,\left\langle\theta_{x}^{2}\right\rangle,\left\langle\theta_{z}^{2}\right\rangle,\left\langle u_{x} \theta_{y}\right\rangle\right)$, the same number as when the expansion of the o.p.d.f. was used [1]. Moreover the values of the reliability factors are comparable. The results are summarized in table I.

\section{Discussion.}

\subsection{ORIENTATIONAL DISORDER.}

4.1.1 Isotropic contribution. - The first major result is that we obtain $p=0$ where $p$ is the weight of the isotropic contribution to the structure factor, and represents the proportion of molecules which are performing random rotations at a given time.

This result is far from trivial as there were many good reasons to expect a non-zero isotropic contribution : i) an incoherent neutron scattering experiment [5] performed on a hydrogenated powdered sample of neopentane had been analysed by using an isotropic two-step stochastic model which combines free rotations and librations. The libration time, i.e.the time between two free rotations which is similar to a residence time, had turned out to vary with temperature from $2.4 \mathrm{ps}$ to $0.3 \mathrm{ps}$. The rotational time can be estimated to vary from $1.5 \mathrm{ps}$ to $1.2 \mathrm{ps}$ in hydrogenated neopentane (Appendix 2). As a consequence, the proportion of free rotating or isotropic molecules varies approximately from 0.4 to 0.8 which is obviously in contradiction with our own results. This will be further discussed in section 3 .

ii) a Raman scattering experiment [6] analysing the spectral profile of an internal mode, had shown very large effects which were attributed to molecular reorientations, thus leading to expect a non-negligible isotropic contribution. Our present results will allow us to criticize this interpretation in section 3 .

iii) another Raman scattering experiment [7] had shown anisotropy effects which were undoubtedly present, but this did not alter our opinion that neopentane is very isotropic, as these effects were very weak. It had been, however, pointed out that it was likely that some averaging had occurred in this experiment because of the presence of several randomly oriented single crystals in the sample.

iv) the whole idea of adding an isotropic contribution to $F^{\mathrm{TLS}}(q)$ had come from a study of

Table I. - Summary of the results, showing the values of the six parameters of the model at the five temperatures on lines 1, 2, 4, 6, 8 and 10. On lines 3 and 5 the translational halfwidths are displayed and on lines 7 and 9 the angular halfwidths converted into degrees. Line 11 shows the weighted reliability factors of each fit, line 12 the number of independent reflections in each experiment, line 13 an average Debye Waller factor to be compared to line 14 the corresponding factor taken from reference [1].

\begin{tabular}{|c|c|c|c|c|c|}
\hline$T$ & $150 \mathrm{~K}$ & $173 \mathrm{~K}$ & $187 \mathrm{~K}$ & $225 \mathrm{~K}$ & $253 \mathrm{~K}$ \\
\hline$F_{\text {norm }}$ & $5.07(0.13)$ & $2.800(0.048)$ & $4.76(0.13)$ & $5.97(0.14)$ & $5.73(0.22)$ \\
$\left\langle U_{x}^{2}\right\rangle / \AA^{2}$ & $0.1091(0.0051)$ & $0.1253(0.0060)$ & $0.162(0.023)$ & $0.1798(0.0053)$ & $0.2132(0.0083)$ \\
$\sqrt{\left\langle U_{x}^{2}\right\rangle / \AA}$ & $0.3303(0.0077)$ & $0.3540(0.0085)$ & $0.403(0.029)$ & $0.4240(0.0062)$ & $0.4617(0.0090)$ \\
$\left\langle U_{z}^{2}\right\rangle / \AA^{2}$ & $0.125(0.016)$ & $0.081(0.017)$ & $0.068(0.057)$ & $0.23(0.020)$ & $0.301(0.036)$ \\
$\sqrt{\left\langle U_{z}^{2}\right\rangle / \AA}$ & $0.354(0.023)$ & $0.285(0.030)$ & $0.26(0.11)$ & $0.480(0.021)$ & $0.549(0.033)$ \\
$\left\langle\theta_{x}^{2}\right\rangle / \mathrm{rd}^{2}$ & $0.0429(0.0045)$ & $0.0552(0.0045)$ & $0.062(0.019)$ & $0.0557(0.0051)$ & $0.0553(0.0080)$ \\
$\sqrt{\left\langle\theta_{x}^{2}\right\rangle / \mathrm{deg}^{2}}$ & $11.87(0.62)$ & $13.46(0.55)$ & $14.3(2.2)$ & $13.52(0.62)$ & $13.47(0.97)$ \\
$\left\langle\theta_{z}^{2}\right\rangle / \mathrm{rd}^{2}$ & $0.0140(0.0032)$ & $0.0141(0.0045)$ & $0.006(0.019)$ & $0.0168(0.0045)$ & $0.0180(0.0079)$ \\
$\sqrt{\left\langle\theta_{z}^{2}\right\rangle / \mathrm{deg}}$ & $6.78(0.77)$ & $6.8(1.1)$ & $4.4(7.0)$ & $7.43(0.99)$ & $7.7(1.7)$ \\
$\left\langle U_{x} \theta_{y}\right\rangle / \AA . \mathrm{rd}$ & $0.0201(0.0030)$ & $0.0207(0.0043)$ & $0.015(0.020)$ & $0.0280(0.0046)$ & $0.0275(0.0088)$ \\
$R W \%$ & 7.5 & 4.8 & 3.3 & 5.5 & 6.9 \\
nbr of reflex. & 32 & 31 & 13 & 27 & 21 \\
{$\left[2\left\langle U_{x}^{2}\right\rangle+\left\langle U_{z}^{2}\right\rangle\right] / 3 / \AA^{2}$} & $0.1144(0.0087)$ & $0.1105(0.0097)$ & $0.131(0.034)$ & $0.197(0.010)$ & $0.243(0.018)$ \\
$\left\langle U_{x}^{2}\right\rangle$ opdf $/ \AA^{2}$ & $0.103(0.004)$ & $0.101(0.07)$ & $0.112(0.01)$ & $0.172(0.005)$ & $0.212(0.006)$ \\
\hline
\end{tabular}


$\mathrm{CBr}_{4}$ [8], another plastic crystal very close to neopentane (same lattice and molecular symmetry, same $D_{2 \mathrm{~d}}$ most probable orientation) in which the TLS model used alone had not supplied good results. A more sophisticated model [9] including an isotropic contribution had been used later and had given $p=0.13$. It should be noted, however, that the electronic cloud of bromine is much larger than that of hydrogen and easily deformed, so that, at very short distance, the $\mathrm{Br}-\mathrm{Br}$ potential, which is not very well known, could be relatively soft. This would lessen, comparatively to neopentane, the steric hindrance to free or isotropic rotations and could explain the difference between the two systems.

In any case, our present result $(p=0)$ implies that plastic neopentane exhibits much less orientational disorder than was deduced from the previous experiments. We must however point out that this does not mean that no reorientations at all occur : uniaxial rotations around given molecular or crystalline axes would contribute very little to the isotropic form factor and would not be detected by the present analysis.

4.1.2 «Librational» amplitude. - The « librational» half-widths turn out to be approximately $13^{\circ}$ and $7^{\circ}$ for $\sqrt{\left\langle\theta_{x}^{2}\right\rangle}$ and $\sqrt{\left\langle\theta_{z}^{2}\right\rangle}$ respectively, with a very small thermal dependence. These values, along with the fact that the isotropic term is zero, explain the origin of the difficulties encountered with the o.p.d.f. expansion analysis :

i) the angular resolution of the highest order symmetry adapted function which could reasonably be used, $R_{11}^{10}(\Omega)$ is $18^{\circ}$, so that the largest oscillations could hardly be detected.

ii) there are large regions of $\Omega$ for which the o.p.d.f., $P(\Omega)$, is close to zero ; this is very difficult to account for with such an expansion, as it is the case when expanding with a finite number of terms, a step function in a Fourier series for instance.

It follows that the o.p.d.f. expansion could not give any precise information about the orientational disorder, apart from the most probable orientation. The claim, however, made in [1], that many of the molecules are not in their most probable orientations, which contradicts apparently point ii), is not disproved by the present work. The angular halfwidths $\sqrt{\left\langle\theta_{x}^{2}\right\rangle}$ and $\sqrt{\left\langle\theta_{z}^{2}\right\rangle}$ are large enough to lessen significantly the probability that a molecule is strictly in a $D_{2 \text { d }}$ orientation.

The word librational, which originates from the TLS model (a model meant to deal with ordered molecular crystals), can be misleading (hence the quotes above). Indeed, the large value obtained for $\sqrt{\left\langle\theta_{x}^{2}\right\rangle}$ could have resulted from the fact that the equilibrium orientations are slightly atilt from the pure $D_{2 \mathrm{~d}}$ orientations and not from a large librational motion. This was checked by allowing, in the fit, the most probable orientations (which in a first step were assumed to be strictly $D_{2 d}$ ) to be rotated around the $x$ axis (Fig. 1), then providing 24 distinct orientations instead of 6 . Doing this did not change the results below $200 \mathrm{~K}$ as the tilt angle was found to be equal to zero. For the highest two temperatures, $225 \mathrm{~K}$ and $253 \mathrm{~K}$, this angle was found to be approximately equal to $8^{\circ}$ and $12^{\circ}$, i.e. roughly the same value as $\sqrt{\left\langle\theta_{x}^{2}\right\rangle}\left(12^{\circ}\right.$ and $11^{\circ}$ respectively) with only a small improvement in the quality of the fit. Such a result does not favor a tilted orientation of the molecule, but rather suggests that the probability distribution is no longer Gaussian at these temperatures.

\subsection{TherMal EVOLUTION.}

4.2.1 Angular variables. - Most previous experiments $[5,7,10]$ had concluded in a rapid variation of the orientational disorder with temperature. As $p$ remains equal to zero at all temperatures, one would expect this disorder to show on the thermal variations of $\left\langle\theta_{x}^{2}\right\rangle$ and $\left\langle\theta_{z}^{2}\right\rangle$ (Fig. 2). This however is not the case.

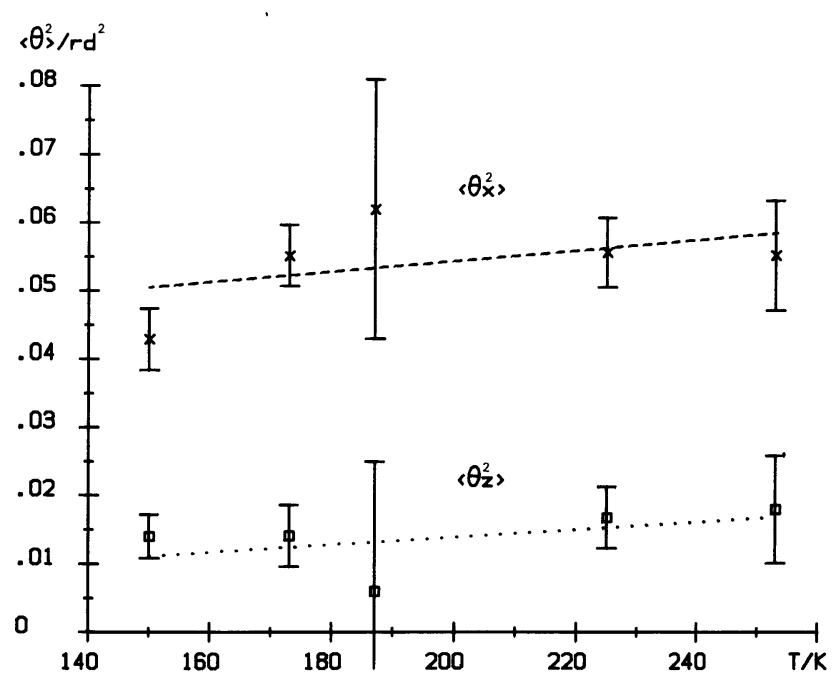

Fig. 2. - Variation of the angular variables with temperature. Crosses : $\left\langle\theta_{x}^{2}\right\rangle$; open squares : $\left\langle\theta_{z}^{2}\right\rangle$.

On the other hand, one may compare our results to a purely librational model :

$$
\frac{1}{2} k_{\mathrm{B}} T=\frac{1}{2} I \dot{\theta}^{2}=\frac{1}{2} I \omega^{2}\left\langle\theta^{2}\right\rangle
$$

where $\dot{\theta}$ is the angular velocity and $\omega$ the librational frequency.

The frequency $\omega$ is unknown but, assuming that $\omega$ remains constant, equation (5) implies that $\left\langle\theta^{2}\right\rangle$ is proportional to the temperature, which is by no 
means the case. This clearly shows that there is a large non-librational contribution to $\left\langle\theta_{x}^{2}\right\rangle$ and $\left\langle\theta_{z}^{2}\right\rangle$, i.e. the molecules are distributed around their most probable orientations. Two different interpretations may be suggested :

i) the individual equilibrium orientations for each molecule may be distributed around the $D_{2 \mathrm{~d}}$ orientations causing an additional static disorder,

and ii) the potential minima may be almost flat allowing the molecules to rotate more or less freely within certain angular limits (which may vary from site to site) around the $D_{2 \text { d }}$ orientations, in which case the disorder would be dynamical without causing a proportionality to the temperature.

The actual case presumably lies in between those two extreme situations but an elastic scattering experiment cannot help deciding on this point.

\subsubsection{Translational motions. - Figure 3 displays the} comparison of $\frac{2\left\langle u_{x}^{2}\right\rangle+\left\langle u_{z}^{2}\right\rangle}{3}$ obtained in the present work and $\left\langle u^{2}\right\rangle_{\text {o.p.d.f. }}$ from the o.p.d.f. analysis, showing a good overall agreement between both models, with however a small systematic difference as $\left\langle u^{2}\right\rangle_{\text {o.p.d.f. }}$ is always slightly smaller, which could be caused by the absence of bond length corrections in our model.

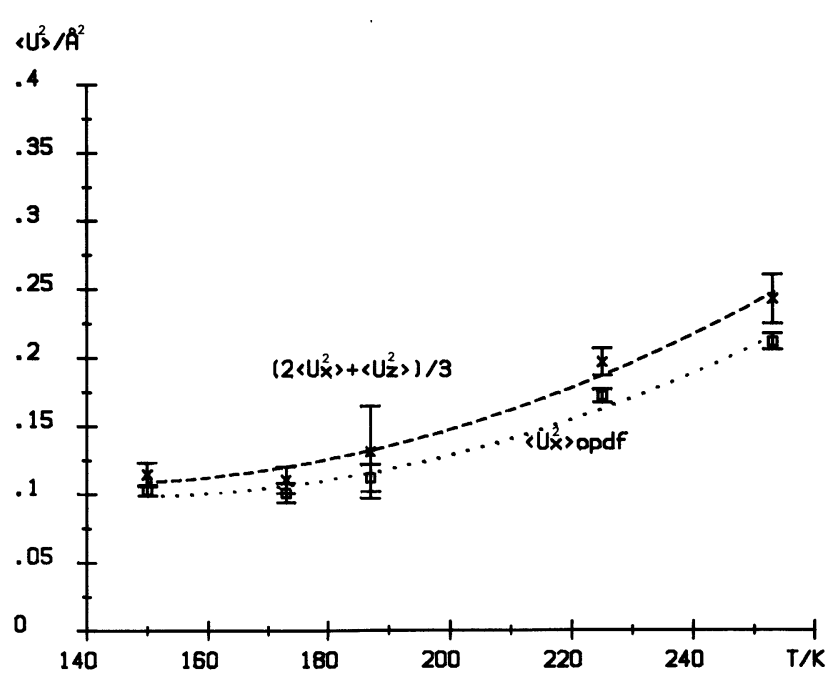

Fig. 3. - Comparison between the Debye Waller factor in both models ; crosses : $\frac{\left(2\left\langle u_{x}^{2}\right\rangle+\left\langle u_{z}^{2}\right\rangle\right)}{3}$ from this work ; open squares : $\left\langle u_{x}^{2}\right\rangle_{\text {o.p.d.f. }}$ from the o.p.d.f. analysis. The lines are fitted $2^{\text {nd }}$ degree polynomials.

The TLS model supplies additional information, as $\left\langle u_{x}^{2}\right\rangle$ and $\left\langle u_{z}^{2}\right\rangle$ are different and display different thermal evolutions (Fig. 4).

$\left\langle u_{x}^{2}\right\rangle$ increases linearly at the rate of $10^{-3} \AA^{2}$ $\mathrm{K}^{-1}$, much faster than what a Debye model would

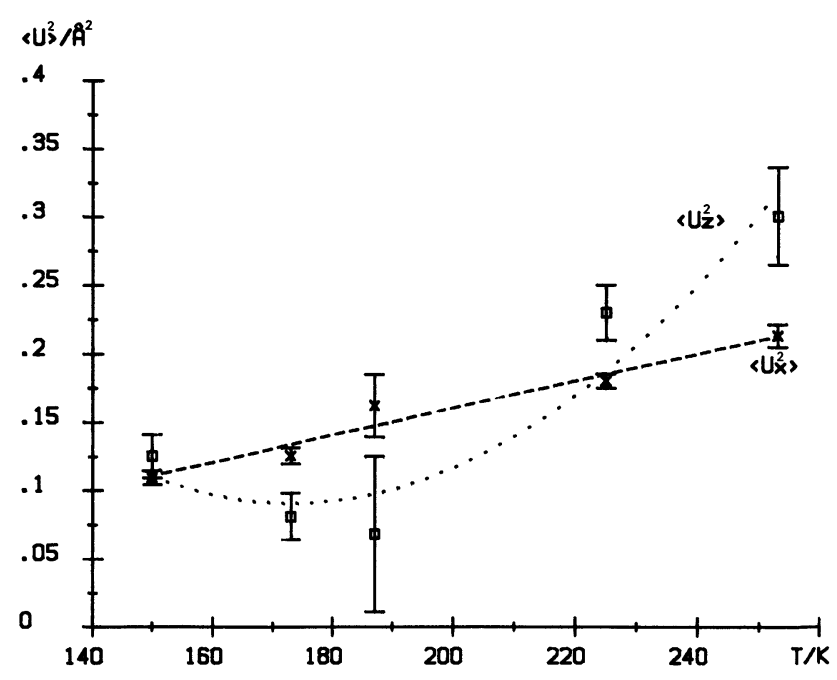

Fig. 4. - Variation with temperature of the translational variables, crosses : $\left\langle u_{x}^{2}\right\rangle$; open squares : $\left\langle u_{z}^{2}\right\rangle$. The dotted line is a $2^{\text {nd }}$ degree polynomial.

predict $\left(0.25 \times 10^{-3} \AA^{2} \mathrm{~K}^{-1}\right)$. This suggests that as pointed out in [1], because of the steric hindrance, there is a correlation between the orientation of one molecule and the centre-of-mass displacements of the neighbouring molecules, so that the increase of rotational motion, which is not visible on rotational variables because of the small number of rotating molecules, nevertheless has a definite influence on the translational disorder. This may be understood as the reorientations of one molecule is likely to affect the centre-of-mass position of most, if not of all, the twelve neighbouring molecules.

In the high temperature region, $\left\langle u_{z}^{2}\right\rangle$ increases at an approximate rate of $3 \times 10^{-3} \AA^{2} \mathrm{~K}^{-1}$. But very surprisingly $\left\langle u_{z}^{2}\right\rangle$ does not increase between $150 \mathrm{~K}$ and $173 \mathrm{~K}$ (the uncertainty is too large at $187 \mathrm{~K}$ to conclude at that temperature). This would be strictly impossible if $\left\langle u_{z}^{2}\right\rangle$ resulted only from a translational phonon contribution. As with $\left\langle\theta_{x}^{2}\right\rangle$, we attempted to find out whether this could be caused by the fact that the equilibrium position might be displaced by $\delta_{z}$. The answer is again unconclusive : no improvement of the reliability factor was obtained, the fit program being unable to choose between $\delta_{z}=$ 0 and $\left\langle u_{z}^{2}\right\rangle \neq 0$, on the one hand, and $\delta_{z} \neq 0$ and $\left\langle u_{z}^{2}\right\rangle=0$ on the other hand.

4.3 DISCUSSION OF PREVIOUS EXPERIMENTAL RESULTS. - Although we cannot conclude on the static or dynamical origin of the $T$ and $L$ factors and on some aspects of their thermal evolution, it is now possible to understand why the present results disagree so much with the previous interpretations of experimental results.

The Raman scattering experiment [6] was performed on an internal vibrational band $\left(922 \mathrm{~cm}^{-1}\right)$ which had, along with the rotational contribution, an 
inhomogeneous broadening contribution. The latter was assumed to be constant as temperature increased, with seemingly good reason at the time, since the bandwidth did not change at the transition at $140 \mathrm{~K}$ from the low temperature ordered phase to the ODIC phase. However, the $922 \mathrm{~cm}^{-1}$ band originates from an internal mode which is both Raman and infrared active [11] which implies the presence of induced dipole-induced dipole (IDID) interaction between the modes on different molecules with an $r^{-3}$ [13] dependence on the intermolecular distance. The inhomogeneous broadening visible in the Raman spectra, caused by the local environment distribution, is thus most probably linked to the IDID interactions and therefore very sensitive, through the $r^{-3}$ term, to the distribution of intermolecular distances. Or the rapid thermal evolution of the Debye Waller factors means that the translational disorder increases rapidly with temperature so that the intermolecular distance distribution also varies significantly, finally resulting in a variation of the inhomogeneous broadening, in contradiction with our first assumption in [6].

Two incoherent neutron scattering experiments had been performed but analysed in a different way. The first, already mentioned [5], considered an isotropic rotational model, completely neglecting the crystal field on one molecule due to its neighbours. The second [10] took into account this crystal field, correctly deduced a $D_{2 \mathrm{~d}}$ mean orientation, and predicted a reorientational mechanism through an instantaneous jump model, thus not taking into account the reorientational trajectories. However in both cases, the classical separation of rotational and translational variables had been performed, as there was, at the time, no reason not to do so. At present, since the thermal behaviour of the translational variables cannot be accounted for by a translational phonon contribution, there is reason to believe that the translational and rotational variables are correlated. This will affect of course the separation of the translational and rotational contributions to the spectra, but also the proton distances to the lattice sites will not be the same before and after a reorientation, and this effect will increase with temperature, as the Debye Waller factor does. This means that the thermal evolution of the residence times for instance may be somewhat incorrectly predicted by these previous analyses.

4.4 Rotation-TRANSLATION COUPLING. - The pertinent parameter in the TLS model is $\left\langle u_{x} \theta_{y}\right\rangle=$ $\left\langle u_{y} \theta_{x}\right\rangle$ which is the correlation for one molecule between its centre-of-mass translations and its rotations around axes in the $(x, y)$ plane.

The ratio $\left\langle u_{x} \theta_{y}\right\rangle / \sqrt{\left\langle\theta_{x}^{2}\right\rangle}$ turns out to be roughly equal to $0.1 \AA$ and constant as temperature increases. This gives the correlated displacement of the molecular centre-of-mass for an average rotation. It is by no means negligible as it represents $1 / 4$ to $1 / 3$ of $\sqrt{\left\langle u_{x}^{2}\right\rangle}$. On the other hand the introduction of the coupling improves the reliability factor by about $1 \%$ which is not drastic.

This is however only part of the problem, as this term only describes the correlation between the translations and the «librations » $\left({ }^{2}\right)$ of the same molecule. As already pointed out, two other contributions certainly play an important role but cannot be taken into account in the present analysis :

i) the correlation between the rotation of one molecule with the centre-of-mass displacement of neighbouring molecules which we have no direct access to, at present, although it is strongly suspected from the thermal behaviour of the translational variables,

and ii) the translation configuration coupling : we have no proof that the centres-of-mass equilibrium position is the same in the six most probable orientations, since the attempt to shift the centre-ofmass position in 4.2.2 was unconclusive.

\section{Conclusion.}

A very classical and fairly unsophisticated model has been used to describe a situation for which it is $a$ priori not well adapted. It supplies surprisingly as good fits as the expansions of the o.p.d.f. on a set of symmetry adapted functions, a model especially meant to deal with such cases. Moreover, it provides information which is much easier to understand :

- the orientational probability function is very anisotropic contrary to expectations, which explains why the o.p.d.f. expansion is unsatisfactory. The probability for a molecule to be far from one of its six most probable $D_{2 \mathrm{~d}}$ orientations is practically zero at all temperatures, and this orientational probability is quite unsensitive to thermal effects, contrary to what had been concluded from previous experiments ;

- both the angular and translational displacements are anisotropic: $\left\langle u_{x}^{2}\right\rangle$ is different from $\left\langle u_{z}^{2}\right\rangle$ and $\left\langle\theta_{x}^{2}\right\rangle$ from $\left\langle\theta_{z}^{2}\right\rangle$;

- non-dynamical effects are suspected for both : neither the « librational » widths can be fully explained by a librational contribution, nor the translational widths by a translational phonon contribution ;

- the problem of the rotational-translational coupling is complex, as it contains the individual translation libration coupling, but also the coupling between the orientation of one molecule and the translation of its neighbours.

It is quite probable that more sophisticated models, including for instance anharmonicity, would

$\left({ }^{2}\right)$ Same restriction as in 4.1.2 about the word «libration ». 
provide better reliability factors, at the cost of an increased number of parameters, but it seems that most of the pertinent information has been extracted through the very simple TLS analysis.

\section{Appendix 1.}

In what is referred to in the present paper as the o.p.d.f. analysis or o.p.d.f. expansion, $P(\Omega)$ is the probability for a molecule to have the orientation $\Omega$ relative to a crystal reference frame, where $\Omega$ is a set of Euler angles. This orientational probability is written as :

$$
P(\Omega)=\sum_{l, \lambda, \lambda^{\prime}} \frac{2 \ell+1}{8 \pi^{2}} A_{\lambda \lambda^{\prime}}^{\ell}, R_{\lambda \lambda^{\prime}}^{\ell}(\Omega)
$$

where $R_{\lambda \lambda^{\prime}}^{\ell}(\Omega)$ is a symmetry adapted function, $\ell$ is related to the number of nodes of $R_{\lambda \lambda^{\prime}}^{\ell}(\Omega)$ as in the case of spherical harmonics $Y_{m}^{\ell}(\theta, \phi)$, and $\lambda$ (resp. $\left.\lambda^{\prime}\right)$ labels the irreducible representations of the site (resp. molecular) symmetry point group.

It has been demonstrated [2] that the $A_{\lambda \lambda^{\prime}}^{\ell}$ coefficients of this expansion can be made to appear in the structure factor; so they can be obtained in a fit allowing $P(\Omega)$ to be computed.

The main assumptions of the model are :

1) The molecules are assumed to be rigid bodies.

2) The o.p.d.f. is assumed to be fairly isotropic, so that the high order coefficients which describe rapid variations will be negligible. In the case of neopentane, the expansion was truncated after $\ell=10$, so that the number of parameters in the fit (six : $F_{\text {norm }},\left\langle u_{x}^{2}\right\rangle, A_{11}^{4}, A_{11}^{6}, A_{11}^{8}, A_{11}^{10}$ ) would remain reasonable compared to the amount of available data (approx. 30 independent reflexions in the best cases). Many truncation artefacts had shown up during the analysis (negative values for $P(\Omega)$, violent non-significant oscillations) making the interpretation of details impossible.

\section{Appendix 2.}

The duration of a molecular reorientation can be estimated by assuming $90^{\circ}$ rotations around a $\overline{4}$ molecular axis with an angular velocity equal to its thermal mean value $: \sqrt{\frac{k_{\mathrm{B}} T}{I}}$ where $I$ is the molecular moment of inertia. This yields :

$$
\tau_{\text {reor }}=\frac{\pi}{2} \sqrt{\frac{I}{k_{\mathrm{B}} T}} .
$$

$I$ was calculated with $r_{1}=1.58 \AA$ and $r_{2}=$ $2.17 \AA$, the values in reference [1] where no apparent bond length shortening appears as in the present article.

$I$ is equal to $2.58 \times 10^{-45} \mathrm{~kg} \mathrm{~m}^{2} \quad(1.95 \times$ $10^{-45} \mathrm{~kg} \mathrm{~m}^{2}$ in the case of an hydrogenated molecule $\left.\mathrm{C}\left(\mathrm{CH}_{3}\right)_{4}\right)$, so that $\tau_{\text {reor }}$ varies from approximately $1.8 \mathrm{ps}$ at $150 \mathrm{~K}$ to $1.4 \mathrm{ps}$ at $253 \mathrm{~K}$ (respectively $1.5 \mathrm{ps}$ and $1.2 \mathrm{ps}$ in $\left.\mathrm{C}\left(\mathrm{CH}_{3}\right)_{4}\right)$.

Another possible evaluation of $\tau_{\text {reor }}$ is to assume that the kinetic energy of a reorienting molecule is not the ensemble average, but the hindering barrier i.e. approximately $500 \mathrm{~K}[10]$. This supplies $\tau_{\text {reor }}=$ $1 \mathrm{ps}$ for the deuterated molecule, and $0.8 \mathrm{ps}$ for the hydrogenated molecule.

However, computer simulations [14] show that the actual rotational motions are extremely complex, so that neither estimate is really correct, but the order of magnitude is sufficient for our purpose.

\section{References}

[1] Denise, B., Depondt, Ph., Debeau, M. and SCHweIss, P., J. Physique 48 (1987) 615.

[2] Press, W. and Hüller, A., Acta Crystallogr. A 29 (1973) 252.

[3] Willis, B. T. M. and Pryor, A. W., Thermal vibrations in crystallography (Cambridge University Press) 1975, chap. 6.

[4] Shomaker, V. and Trueblood, K. N., Acta Crystallogr. B 24 (1968) 63.

[5] Larsson, K. E., MÅnsson, T. and Olsson, L.G., International Symposium on Neutron Inelastic Scattering, Vienna (1977).

[6] Depondt, Ph., Debeau, M. and Pick, R. M., J. Chem. Phys. 77 (1982) 2779.
[7] Depondt, Ph. and Debeau, M., J. Chem. Phys. 77 (1982) 30 .

[8] More, M., Thèse U.S.T. Lille (1982).

[9] Hohlwein, D., Z. Kristallogr. 169 (1984) 237.

[10] Steenbergen, Chr. and de Graaf, L. A., Physica 96B (1979) 1.

[11] Livingston, R. C., Rothschild, W. G. and Rush, J. J., J. Chem. Phys. 59 (1973) 2498.

[12] Durig, J. R., Craven, S. M. and Bragin, J., J. Chem. Phys. 51 (1969) 5663.

[13] BreymanN, W., Yvinec, M. and Pick, R. M., J. Chem. Phys. 82 (1985) 5318.

[14] BREYMANN, W., to be published. 